\title{
Circuit
}

Musiques contemporaines

\section{Bruit, son, silence : silence et liturgie}

\section{Denise Robillard}

Volume 5, numéro 1, 1994

Gilles Tremblay : réflexions

URI : https://id.erudit.org/iderudit/902089ar

DOI : https://doi.org/10.7202/902089ar

Aller au sommaire du numéro

Éditeur(s)

Les Presses de l'Université de Montréal

ISSN

1183-1693 (imprimé)

1488-9692 (numérique)

Découvrir la revue

Citer cet article

Robillard, D. (1994). Bruit, son, silence : silence et liturgie. Circuit, 5(1), 46-50. https://doi.org/10.7202/902089ar d'utilisation que vous pouvez consulter en ligne.

https://apropos.erudit.org/fr/usagers/politique-dutilisation/ 


\section{Silence et liturgie}

I Propos recueillis par Denise Robillard pour Liturgie, foi et culture, 1989.

Deux aspects du silence sont liés à la liturgie: le silence dans le temps et le silence dans l'espace. Je me suis souvent arrêté à la question du silence dans le temps, car pour un musicien, le silence, c'est en quelque sorte le matériau premier, le matérieu indispensable à la naissance de la musique.

\section{Le temps du silence}

Techniquement, on peut considérer le silence selon trois zones du temps: le silence qui est "avant», le silence qui est «pendant» et le silence qui est «après». Le silence «avant», c'est le silence qui prépare une liturgie, une prière, une expression quelconque de parole, une lecture de psaume ou 
d'évangile. Cela se comprend très bien, c'est comme une espèce de respiration avant $d^{\prime}$ 'aborder un texte. Le silence "après» est aussi facile à comprendre: c'est une espèce de reflux des pensées qui ont été remuées. Le silence répond au besoin de temps pour méditer ces pensées.

Mais ce qui paraît moins évident, c'est le silence «pendant», qui est le silence de l'écoute totale, de l'écoute adhésion. Pendant ce temps, on ne fait plus de bruit avec sa propre pensée. C'est vrai pour la liturgie et c'est aussi une expérience musicale, c'est vrai aussi bien pour le son que pour la parole. J'irais plus loin en ce qui concerne la musique : même «pendant " le son, il y a du silence, car le silence n'est pas uniquement une absence de son, un silence négatif.

\section{Présence et écoute}

Le vrai silence a une signification beaucoup plus profonde que l'absence de son. Le vrai silence peut être tout autant présence qu'absence. Une présence de clarté, un peu comme une eau qui, après avoir été agitée, permet d'observer les poussières et les particules en suspension; mais dès qu'on cesse d'agiter l'eau, les particules se déposent au fond et l'eau, toujours présente, devient pleinement transparente, invisible, mais présente.

Dans le prolongement du silence comme transparence, on peut parler du silence «écoute». Un silence actif. Dans la liturgie, si les silences sont des points morts, ils ont raté leur rôle. Tous les mystiques en ont parlé - et la musique est une démarche analogue à la mystique - il n'y a pas d'écoute possible, de prière possible, sans ce vide silencieux, un silence d'épanouissement. II y a des silences amicaux, bienveillants, mais il y a aussi des silences oppressifs. Quand on parle de l'Église du silence, ou quand deux personnes sont en colère, on ne parle pas d'un silence souhaitable! II y a des silences qui sont pires que des cris.

C'est l'expérience du compositeur. Au début d'une œuvre musicale et même de toute idée, il y a un silence nécessaire à la pensée. La pensée naît dans le silence.

\section{L'espace du silence}

L'idée de l'espace du silence me paraît particulièrement importante à l'heure actuelle. Alors qu'on s'interroge sur le rôle de l'église bâtiment - on 
a même démoli plusieurs églises -, il me paraît absurde de détruire des espaces de respiration dans les villes. Ils sont nécessaires à la vie d'une société.

On s'oriente vers un monde où de tels espaces vont apparaître de plus en plus nécessaires. On devra même, pour sauvegarder notre équilibre, construire des espaces de silence. C'est ainsi, par exemple, que depuis longtemps on aménage des parcs. De tels espaces de silence qui sont des espaces de prière, me paraissent aujourd'hui d'une nécessité plus urgente qu'à n'importe quelle autre époque. Cela fait partie d'un patrimoine - le patrimoine du silence - et on devrait tenir comme à la prunelle de ses yeux à certains espaces de silence!

Dans la liturgie proprement dite, - et ici, c'est le citoyen ordinaire qui transmet ce qu'il ressent -, il arrive qu'on participe, avec les mêmes personnes ou les mêmes communautés, à des liturgies qui sont bien ou moins bien réussies. Une question de grâce ?... ou de préparation ? $\|$ y a des sommets de la liturgie eucharistique qui sont trop souvent escamotés. Est-ce en raison de courants théologiques ou de psychologies à la mode qu'on en est venu à araser certains sommets?

Un de ces sommets, c'est la consécration. J'éprouve chaque fois le même malaise quand, après la consécration, on ne ménage pas un moment d'adoration et de silence avant d'entonner l'acclamation. Ce n'est pas très naturel, ni spirituellement ni même esthétiquement. II y a d'autres sommets, comme la doxologie précédant le Pater, qui méritent un silence...

En musique, il y a des auteurs qui utilisent le silence avec plus de bonheur que d'autres. Mozart, par exemple. Sa musique tellement vivante, est toute pleine de silence. II sait avec génie mettre le silence là où il faut. Dans la liturgie, il existe un problème de forme, un problème technique, sur lequel les personnes qui préparent la liturgie devraient se pencher. II ne s'agit pas de parsemer la liturgie de silences qui en arrêteraient le mouvement, mais au contraire, avec des silences qui n'ont pas besoin d'être très nombreux, on pourrait réussir à faire ressortir le mouvement de la liturgie.

\section{Une question de qualité}

Je ne pense pas qu'il faille faire de la liturgie une espèce d'horlogerie à la suisse où toute serait calculé au quart de seconde. Non. C'est plus simple que cela. Le paradoxe, c'est qu'il faut que la liturgie soit très bien structurée, 
sans que cette structure ne soit directive. Si la liturgie est bien structurée dans son ensemble, la collectivité pourra davantage prier à son rythme. C'est beaucoup plus qualitatif que quantitatif.

C'est le contexte, ce qui a été dit dans I'homélie, ce qui est vécu, qui inspirera certains jours des silences plus longs parce qu'ils auront été plus nourris. A mon avis, s'il y avait une écoute plus grande de la part de la communauté, cela pourrait se faire spontanément.

Mais la meilleure façon, pour les responsables de la liturgie, de se préparer à aménager des silences au cours d'une célébration, c'est de pratiquer eux-mêmes le silence, c'està-dire de prier! On éprouve trop souvent une certaine pudeur, quand il s'agit de prendre des décisions au moment de la préparation de la célébration, à réagir spontanément par la prière. On a plutôt tendance à meubler les silences au lieu de les écouter.

Nous sommes envahis par les bruits. C'est une des pollutions du monde moderne. Il faut en être conscient et la dénoncer. C'est là l'aspect négatif du bruit.

La prospective positive consiste dans l'écoute attentive des bruits du monde moderne qui permet de découvrir des courbes de beauté incroyables. Cela reste contradictoire, car on peut trouver esthétique le bruit d'un avion qui passe, alors que la personne habitant dans le prolongement de la piste d'aviation aura une autre perception des mêmes bruits qui troublent son sommeil! II reste que l'acceptation amicale du monde moderne nous aide à vivre harmonieusement, mais il faut maîtriser le flot de bruits qui nous envahit.

La liturgie pourrait être un moment pour s'y exercer, mais comment $\zeta \|$ faudrait peut-être en faire un offertoire, ce moment où, traditionnellement, on apporte des biens devant l'autel. On s'apporte soi-même avec le monde dans lequel on vit, avec le fatras de préoccupations qui nous habitent, avec le désordre et l'agitation qui sont en nous. On dépose son être tel qu'il est, sans cacher quoi que ce soit.

La pratique du silence dans la liturgie, l'offrande de notre agitation me rappellent la parole de François d'Assise que je paraphrase: a La beauté de Dieu est plus grande que toute la laideur possible ». Quand nous venons prier, dans ce geste de déposer, nous vivons l'expérience du désencombrement.

Les attitudes du célébrant et des intervenants sont essentielles pour entraîner le peuple dans cette expérience. C'est dans l'esprit que se fait cet offertoire ef il faut s'éduquer soi-même à le faire simplement. Quand une zone de 
silence précède une prière, on a l'impression que la prière qui suit est plus forte, plus pleine.

$\mathrm{Si}$, dans nos liturgies, certains moments sont très bien réussis, en général, les moments forts ne sont pas suffisamment mis en valeur par cette espèce d'écrin de silence. Comme si l'on avait peur du silence.

\section{Le choc du silence}

La liturgie ne doit pas se laisser gagner par la philosophie de la musique MUSAK, une musique qui meuble. Comment «gérer» le silence? Le mot " gérer * doit être entendu au sens qualitatif. Nous sommes tous en mesure de faire une expérience de silence extraordinaire, soit dans un monastère, soit dans un groupe de prière: le début est toujours pénible, on éprouve un malaise, on a envie de meubler le silence. Mais si l'on passe ce cap, si on franchit ce mur, on est nourri, tout devient aisé, on finit par se demander comment on peut vivre sans pratiquer ce silence. Les liturgies et l'espace des églises, même en dehors de la liturgie, sont des occasions de passer à travers ce mur. Le silence a un rôle éducateur, on peut parler d'une pédagogie du silence.

Ce qui aide à la qualité du silence, c'est la qualité des textes liturgiques eux-mêmes. Ils rayonnent en deux branches: la branche silencieuse et la branche musicale. Le lien entre la musique et le silence dans la liturgie est presque co-naturel, parce que dans la musique, il y a aussi une part de méditation et une part de silence, successives l'une à l'autre. Le silence «avant * prépare très bien à la musique, mais la musique prépare aussi très bien au silence.

Par goût personnel, j'estime que le moment de l'action de grâce après la communion devrait comporter un espace de recueillement. Ce n'est pas le moment de chanter. Mais quels que soient les tempéraments, les charismes ou les états d'âme, cette « gestion », cet « aménagement * du silence mérite qu'on s'y arrête, exige qu'on se donne des repères. C'est une question de qualité, cela ne peut être improvisé. II faut préparer, prévoir, pour ensuite aménager selon le besoin. Les intervenants, en particulier le célébrant, devraient avoir une expérience vécue de cette nécessité. Certains le sentent très bien, mais peut-être n'osent-ils pas suffisamment écouter leur instinct. 